\title{
Monetary Policy and the Yield Curve
}

\author{
Antulio N. Bomfim*
}

Federal Reserve Board

First Draft: November 8, 2002

This Draft: February 13, 2003

\begin{abstract}
This paper examines the empirical properties of a two-factor affine model of the term structure of interest rates, estimated with LIBOR and interest rate swap data from 1989 through 2001. Despite its relative simplicity, the model fits the interest rate data remarkably well, both across time and maturity, and identifies changes in the current and expected stance of monetary policy as primary movers of the yield curve.
\end{abstract}

JEL Classification: G12, E43

Keywords: Affine models, expectations hypothesis, latent factors

*Board of Governors of the Federal Reserve System, Washington, DC 20551; E-mail: abomfim@frb.gov; Fax: (202) 452-2301, Tel.: (202) 736-5619. I am grateful to Brian Sack and Hao Zhou for helpful comments, to Joseph Rosenberg for excellent research assistance, and to seminar participants at the Federal Reserve Board for their insights. The opinions expressed in this paper are not necessarily shared by the Board of Governors of the Federal Reserve System or any other members of its staff. 


\section{Introduction}

A growing literature has suggested that the entire yield curve moves primarily in response to changes in a small number of potentially unobservable common factors, but the factors themselves are typically described based on their effects on the "level, slope, and curvature" of the yield curve, rather than in terms of the financial and economic fundamentals that drive market interest rates -Dai and Singleton (2000) and Litterman and Scheinkman (1991). ${ }^{1}$ In this paper, I discuss and test empirically an economic interpretation for an otherwise standard no-arbitrage multifactor affine framework, very much in the tradition of Balduzzi, Das, and Foresi (1998) and Babbs and Nowman (1999). The results suggest that actual and expected changes in monetary policy account for a remarkably large share of the variation in bond yields in the United States across the whole maturity spectrum.

The proposition that monetary policy plays an important role in the determination of market interest rates is hardly new. Indeed, in laying the groundwork for the two-factor affine model used in the analysis, I briefly review, in Sections 2 and 3, a well-known form of the expectations hypothesis of the yield curve, which postulates that yields on longer-term bonds reflect market participants' expectations of future values of short-term yields, which are themselves largely set by monetary policy. What is new and noteworthy among the results reported in this paper is the extent to which estimates

\footnotetext{
${ }^{1}$ Recent work by Ang and Piazzesi (2002) and Piazzesi (2001) deviates somewhat from this norm and is related to this paper to the extent that at least some of the common factors discussed in those papers are given an explicit economic interpretation. For instance, Ang and Piazzesi propose a multifactor framework that incorporates both observable (macroeconomic) factors and latent factors, although the latter are not fully accounted for by identifiable fundamentals.
} 
of the two latent factors that drive the yield curve in the model can be mapped into commonly used measures of the current and expected stance of monetary policy. Based on the estimation approach described in Section 4, I report, in Section 5, that while the first factor tracks observed short-term interest rates very well, the second moves nearly one-to-one with mediumterm monetary policy expectations, as inferred by eurodollar futures rates. The close relationship between the second factor and policy expectations is especially remarkable given that futures rates were not used in the estimation of the model. In Section 6, I discuss the main conclusions of the analysis and point to potential directions for further research.

\section{Background and Motivation}

Let $Z(t, T)$ denote the time- $t$ price of a zero-coupon bond that matures at time $T$ with no risk of default. Assuming, without loss of generality, that the bond has a face value of $\$ 1$, its continuously compounded yield-to-maturity, $R(t, T)$, is such that:

$$
Z(t, T)=e^{-R(t, T)(T-t)}
$$

The continuously compounded time- $t$ forward rate for the future period $\left[T_{1}, T_{2}\right]$ can be shown to be

$$
R\left(t, T_{1}, T_{2}\right)=-\frac{\log \left(Z\left(t, T_{2}\right)\right)-\log \left(Z\left(t, T_{1}\right)\right)}{T_{2}-T_{1}}
$$


and thus the instantaneous forward rate with maturity $T$ - the time- $t$ forward rate for the infinitesimal future period $[T, T+d t]$ - is written as

$$
f(t, T)=-\frac{\partial \log (Z(t, T))}{\partial T}
$$

The instantaneous riskless spot rate, also referred to in this paper as the short rate, is given by:

$$
r(t)=f(t, t)
$$

Note that by integrating both sides of (3) and combining the result with (1) we obtain

$$
R(t, T)=\frac{\int_{t}^{T} f(t, S) d S}{T-t}
$$

which gives the familiar result that the time- $t$ yield on a longer-term zerocoupon bond can be thought of as the average of time- $t$ forward rates prevailing over the life of the bond.

Intuition and financial reasoning tell us that forward rates should embody expectations of future spot rates (plus a potentially non-zero risk premium). Motivated by this insight, the modeling approach adopted in this paper starts with a model for the evolution of the short rate, which, in turn leads to the specification of the common factors behind yield curve fluctuations. Before turning to the specifics of the modeling framework, however, we will take a closer look at equation (5), which should help us relate the model presented here to other work in the term structure literature. In addition, I shall argue that equation (5) is a useful tool for adding an economic interpretation to the model. 


\subsection{The Expectations Hypothesis and a Role for Mon- etary Policy}

Equation (5) serves as a basis for a well-known form of the expectations hypothesis of the term structure of interest rates. This version of the hypothesis is based on the assumption that the instantaneous forward rate $f(t, S)$ is equal to the expectation of the corresponding future spot rate plus a time-invariant risk premium $\phi(S-t)$,

$$
f(t, S)=E_{t}[r(S)]+\phi(S-t)
$$

where $E_{t}[$.$] denotes an expectation conditioned on time- t$ information, computed under the objective probability measure. ${ }^{2}$

Combining equations (5) and (6), we obtain

$$
R(t, T)=\frac{\int_{t}^{T} E_{t}[r(S)] d S}{T-t}+\Phi(T-t)
$$

which is the so-called yield-to-maturity form of the expectations hypothesissee, e.g., Cox, Ingersoll, and Ross (1981)-augmented with a time-invariant risk premium. Equation (7) says that yields on longer-term zero-coupon bonds represent market participants' expectations of the average level of short-term interest rates over the maturity of the bond plus a risk premium $\Phi(T-t) \equiv \frac{\int_{t}^{T} \phi(S-t) d S}{T-t}$.

Equation (7) provides a direct link between longer-term interest rates and

\footnotetext{
${ }^{2}$ In its "pure" version, this form of the expectations hypothesis assumes that $\phi(S-t)=$ 0 . In this paper, I work with the weaker form of the hypothesis, which allows for non-zero risk premiums.
} 
expectations of future short-term interest rates. A common approach in the economics literature on the term structure of interest rates, which I shall refer to as the "structural approach," is to assume that short rates are primarily determined by monetary policy and then to propose an equation-often referred to as a "monetary policy reaction function" - relating movements in the short rate to changes in key macroeconomic variables that monetary policymakers are assumed to care about. Thus, using model-based projections for such macro variables, which are effectively taken to be the factors behind movements in the yield curve, one can derive the model-implied forecasts of the short rate and assess the model's ability to capture the main sources of variation in longer-term yields. ${ }^{3}$

In this paper, I follow what I shall call a "market-based" approach to estimating (7). Rather than trying to model the behavior of the short rate by assuming a specific reaction function for monetary policymakers, and attempting to forecast the macro variables that enter into that function, I rely almost exclusively on observed interest rates to infer the short rate expectations and associated risk premiums in the minds of financial market participants. Indeed, a key idea behind this paper is to impose a minimum set of assumptions on the evolution of the short rate and to let the whole configuration of market interest rates tell us as much as possible about market participants' attitudes toward risk and their expectations of where the short rate (and potentially the yield curve) is headed.

\footnotetext{
${ }^{3}$ This approach is similar in spirit to the work of Evans and Marshall (1998).
} 


\section{The Modeling Framework}

Consider the following model for the evolution of the short rate, $r(t)$,

$$
\begin{aligned}
& d r(t)=k[\theta(t)-r(t)] d t+v d W_{r}(t) \\
& d \theta(t)=\alpha[\beta-\theta(t)] d t+\eta d W_{\theta}(t)
\end{aligned}
$$

$r(t)$ is assumed to error-correct toward its time-varying central tendency $\theta(t)$ with a mean reversion coefficient $k . \theta(t)$ is also assumed to follow a mean-reverting process, with $\alpha$ denoting its speed of mean reversion and $\beta$ its long-run value. $d W_{r}(t)$ and $d W_{\theta}(t)$ are uncorrelated stochastic shocks (infinitesimal increments to standard Brownian motions) hitting the short rate and its central tendency, respectively, and $v$ and $\eta$ are their volatilities. In terms of the economics of the model, $\theta(t)$ can be thought of as where market participants think the monetary authority will take the short rate in the future, an implicit notion of a policy reaction function, and $\beta$ is the steady-state value of the short rate.

The above model is a two-factor extension of the one-factor model originally proposed by Vasicek (1977) and a variant of the double-mean reverting framework discussed by Babbs and Nowman (1999) and the two-factor model of Balduzzi et al. (1998). Equations (8) and (9) make up a system of stochastic differential equations, which can be solved to obtain

$$
X(T)=e^{-K(T-t)} X(t)+\left(I-e^{-K(T-t)}\right) \Theta+\int_{t}^{T} e^{-K(s-t)} \sigma d W(u)
$$


where $X(t) \equiv[r(t), x(t)]^{\prime}, x(t) \equiv \theta(t)-\beta, K \equiv\left[\begin{array}{ll}k & -k \\ 0 & \alpha\end{array}\right], \Theta \equiv[\beta, 0]^{\prime}, \sigma \equiv$ $\left[\begin{array}{cc}v & 0 \\ 0 & \eta\end{array}\right], I$ is a $2 \mathrm{x} 2$ identity matrix, and $d W(u)$, defined as $\left[d W_{r}(u), d W_{\theta}(u)\right]^{\prime}$, is a normally distributed vector with zero mean and variance-covariance matrix $V \equiv \int_{t}^{T} e^{-K(T-u)} \sigma \sigma^{\prime} e^{-K^{\prime}(T-u)} d u$. As a result, it can be shown that the conditional expectation of $X(T)$ is given by

$$
E_{t}[X(T)]=e^{-K(T-t)} X(t)+\left[I-e^{-K(T-t)}\right] \Theta
$$

from which the conditional expectation of $r(T)$ easily follows:

$$
E_{t}[r(T)]=e^{-k(T-t)} r(t)+\frac{k}{k-\alpha}\left[e^{-\alpha(T-t)}-e^{-k(T-t)}\right] x(t)+\left(1-e^{-k(T-t)}\right) \beta
$$

\subsection{A Two-factor Characterization of the Yield Curve}

Given the short-rate model, and assuming that unanticipated movements in $r(t)$ and $\theta(t)$ account for the stochastic variation in forward rates, equation (5) would tell us that one can think of $r(t)$ and $\theta(t)$ as being the two common factors driving fluctuations in the entire yield curve. In particular, one can write the time- $t$ price of a $T_{1}$-maturity zero-coupon bond as a yet to-bedetermined function of the maturity of the bond and of the factors $r(t)$ and $\theta(t), Z\left(t, T_{1}, r(t), \theta(t)\right)$. Given the short-rate model, and invoking Ito's Lemma, we can write down the evolution of the price of this bond in terms 
of the following generic stochastic differential equation:

$$
\frac{d Z^{(1)}(t)}{Z^{(1)}(t)}=\mu^{(1)}(t) d t+\sigma_{r}^{(1)}(t) d W_{r}(t)+\sigma_{\theta}^{(1)} d W_{\theta}(t)
$$

where, to simplify notation, I used the superscript (1) to identify variables corresponding to the $T_{1}$-maturity bond.

Equation (13) can be thought of as the realized return on holding a $T_{1^{-}}$ maturity zero-coupon bond from $t$ to $t+d t$. Accordingly, the bond's corresponding holding period return has mean $\mu^{(1)}(t) d t$ and variance $\left[\sigma_{r}^{(1)}(t)^{2}+\right.$ $\left.\sigma_{\theta}^{(1)}(t)^{2}\right] d t$, where $\sigma_{r}^{(1)}(t)$ and $\sigma_{\theta}^{(1)}(t)$ capture the contributions of shocks to $r(t)$ and $\theta(t)$, respectively, to unexpected changes in the price of the bond.

Equation (13) by itself is of little value in the derivation of an explicit bond pricing formula that relates movements in $Z($.$) , or in R($.$) , to changes in$ the factors $r(t)$ and $\theta(t)$. To derive such a formula, I use a standard valuation methodology that follows the spirit of the Black-Scholes approach to pricing calls and puts. ${ }^{4}$

Suppose we want to price the $T_{1}$-maturity bond. I start by setting up a hedged portfolio containing the bond and two other hedging vehicles - e.g., a $T_{2^{-}}$and a $T_{3}$-maturity bond - one for each type of fundamental uncertainty affecting the price of the $T_{1}$-maturity bond. Let $\Pi(t)$ be the time- $t$ value of the hedged portfolio and $\Delta_{i}$ be the number of unities of the $T_{i}$-maturity bond in the portfolio. Thus, we can write the evolution of the value of the

\footnotetext{
${ }^{4}$ See, e.g., Bjork (1998) for a discussion of this valuation approach.
} 
hedged portfolio as

$$
d \Pi(t)=\sum_{i=1}^{3}\left[\Delta_{i} Z^{(i)}(t)\left(\mu^{(i)}(t) d t+\sigma_{r}^{(i)}(t) d W_{r}(t)+\sigma_{\theta}^{(i)}(t) d W_{\theta}(t)\right)\right]
$$

To ensure the absence of arbitrage opportunities, the instantaneous return on the hedged portfolio must be equal to the riskfree rate, and thus we obtain the following conditions, which the dynamics of the hedged portfolio must satisfy if there are no arbitrage opportunities in the economy:

$$
\begin{array}{r}
\sum_{i=1}^{3} \Delta_{i}\left[\mu^{(i)}(t)-r(t)\right]=0 \\
\sum_{i=1}^{3} \Delta_{i} \sigma_{r}^{(i)}(t) Z^{(i)}(t)=\sum_{i=1}^{3} \Delta_{i} \sigma_{\theta}^{(i)}(t) Z^{(i)}(t)=0
\end{array}
$$

and the above will be true provided that, for all $i$, there exist $\tilde{\lambda}_{r}$ and $\tilde{\lambda}_{\theta}$ such that

$$
\mu^{(i)}(t)-r(t)=\tilde{\lambda}_{r} \sigma_{r}^{(i)}(t)+\tilde{\lambda}_{\theta} \sigma_{\theta}^{(i)}(t)
$$

The left-hand-side of the above equation is essentially the ex-ante risk premium associated with holding a $T_{i}$-maturity bond. The equation implies that this premium can be written as a weighted sum of the contributions of each factor to the overall riskiness of the bond, where the weights are $\tilde{\lambda}_{r}$ and $\tilde{\lambda}_{\theta}$. Given that $\tilde{\lambda}_{r}$ and $\tilde{\lambda}_{\theta}$ capture the relative importance of each risk factor in the determination of the overall risk premium associated with the bond, they are commonly referred to as the "market prices of risk" associated with $r(t)$ and $\theta(t)$, respectively. I assume that the market prices of risk are 
proportional to the volatilities of the underlying factors:

$$
\begin{aligned}
& \tilde{\lambda}_{r}=\lambda_{r} v \\
& \tilde{\lambda}_{\theta}=\lambda_{\theta} \eta
\end{aligned}
$$

After some mathematical manipulation, it can be shown that equations (13) and (17) imply that the time-t price of the $T$-maturity bond, $Z(t, T)$, must satisfy the following partial differential equation (PDE):

$Z_{t}+Z_{r}\left[k(\theta(t)-r(t))-\lambda_{r} v^{2}\right]+Z_{\theta}\left[\alpha\left(\beta-\theta(t)-\lambda_{\theta} \eta^{2}\right]+.5\left[Z_{r r} v^{2}+Z_{\theta \theta} \eta^{2}\right]-r Z=0\right.$

subject to the terminal condition $Z(T, T)=1$, where $Z_{i}$ denotes the partial derivative of $Z(t, T)$ with respect to $i$. ( $Z_{i i}$ is the second partial derivative with respect to i.)

The solution to the above PDE can be shown to have the following form

$$
Z(t, T)=e^{-A(T-t)-B(T-t) r(t)-C(T-t) x(t)}
$$

where, in addition to being maturity-dependent, $A(T-t), B(T-t)$, and $C(T-t)$ are functions of the parameters of the short-rate model and of the market prices of risk.

We can use equations (1) and (21) to write:

$$
R(t, T)=\frac{1}{T-t}[A(T-t)+B(T-t) r(t)+C(T-t) x(t)]
$$

and it is straightforward to see that the conditional expectation of $R\left(T_{1}, T_{2}\right)$, 
computed under the objective probability measure for $T_{2}>T_{1}>t$, is given by

$$
E_{t}\left[R\left(T_{1}, T_{2}\right)\right]=\frac{1}{T_{2}-T_{1}}\left[A\left(T_{2}-T_{1}\right)+\left[B\left(T_{2}-T_{1}\right), C\left(T_{2}-T_{1}\right)\right] E_{t}\left[X\left(T_{1}\right)\right]\right]
$$

Equation (22) is the bond pricing equation implied by the model. For given estimates of the model parameters and of the current values of the short rate and its tendency, (22) gives us the theoretical yield on a zero-coupon bond of any maturity. Thus, using (22), we can, in principle, assess how well the two-factor model fits the yield curve over time and across maturities. I say in principle because this exercise requires estimates of not just the model parameters, but also of the factors $r(t)$ and $\theta(t)$, which are assumed not to be directly observed in financial markets.

\subsection{Revisiting the Expectations Hypothesis}

Let us now examine how the two-factor model relates to the version of the expectations hypothesis discussed in section 2. Using equations (3) and (21), the model-implied forward rates can be written as

$$
f(t, S)=A_{S}(S-t)+B_{S}(S-t) r(t)+C_{S}(S-t) x(t)
$$

where $A_{S}(S-t)$ denotes the partial derivative of $A(S-t)$ with respect to $S$,

and $B_{S}($.$) and C_{S}($.$) are analogously defined. After some substitution and$ rearranging we can arrive at

$$
f(t, S)=E_{t}[r(S)]+A_{S}(S-t)-\left[1-e^{-k(S-t)}\right] \beta
$$


where the last two terms provide an explicit functional form for the modelimplied risk premium associated with the forward rate $f(t, S)$. We have thus mapped the two-factor model into the expectation-hypothesis framework. In particular, we can write

$$
R(t, T)=\frac{\int_{t}^{T} E_{t}[r(S)] d S}{T-t}+\frac{A(T-t)+\beta[B(T-t)-(T-t)]}{T-t}
$$

where the last term corresponds to $\Phi(T-t)$ in equation $(7)$.

Note that the expectations hypothesis, as expressed in (7), generally does not ensure the absence of arbitrage opportunities. In the context of the twofactor model described in this paper, the restriction that

$$
\Phi(T-t)=\frac{A(T-t)+\beta[B(T-t)-(T-t)]}{T-t}
$$

does ensure that the theoretical bond prices implied by the model are consistent both with an arbitrage-free framework and the weak version of the yield-to-maturity form of the expectations hypothesis.

\section{Estimation Approach}

I use zero-coupon yields implied by swap rates and short-term LIBOR to estimate the model. These yields are shown in Figure 1. They are based on six- and twelve-month LIBOR and on swap rates corresponding to two-, three-, four-, five-, seven-, and ten-year maturities. ${ }^{5}$ The data are made up

\footnotetext{
${ }^{5}$ To obtain the implied zero-coupon yields, I first computed zero-coupon bond prices from the LIBOR/swap curve using a standard bootstrapping procedure and then generated
} 
of 667 weekly observations, covering the 1989-2001 period. ${ }^{6}$

The estimation approach allows the factors to be potentially unobservable. Thus, I rely on a Kalman-filter-based maximum likelihood method to simultaneously estimate the model parameters and the time series of the two factors. Intuitively, the estimation routine "looks" at actual movements in the term structure of zero-coupon yields and uses statistical criteria to determine which portion of those movements can be ascribed to changes in the factors, where the factors are constrained to evolve according to equations (8) and (9). Formally, the equations that are estimated are

$$
\begin{aligned}
y(t) & =D_{0}(\Omega, \Lambda)+D_{X}(\Omega, \Lambda) X(t)+u(t) \\
X(t+s) & =G_{0}(\Omega)+G_{X}(\Omega) X(t)+e(t+s)
\end{aligned}
$$

where (28), the measurement equation, is simply (22) expressed in matrix form to include $n$ maturities and augmented with maturity-specific measurement error terms. Accordingly, $y(t)$ is an $n$-by- 1 vector of zero-coupon bond yields implied by the LIBOR and swap curves, and $u(t)$ is the corresponding vector of measurement errors - $\mathrm{u}(\mathrm{t})$ is assumed to have zero mean and a diagonal variance-covariance matrix H. Equation (29) - called the transition equation - is simply equation (10) restated in more compact notation, where $e(t+s)$ is a vector of stochastic shocks with variance-covariance matrix $V(\Omega)$. ( $\Omega$ is the vector of parameters of the short rate model, and $\Lambda$ is the vector of market prices of risk.)

a smoothed curve using the cubic hermite interpolation method. (I also experimented with a cubic spline, and the results were largely unaffected.)

${ }^{6}$ The beginning of the sample is dictated by data availability. 
Equations (28) and (29) fit neatly into the standard Kalman filter framework and make it clear why I characterized the modeling approach used in this paper as market-based. Other than assuming that the unobserved common factors follow mean-reverting processes in an economy that is free of arbitrage opportunities, all that I use to jointly estimate the model parameters and the factors are the market-implied yields on zero-coupon bonds of different maturities. I make no particular assumptions about the form of the policy reaction function behind movements in short rates or about the shape of investors' utility functions and their attitudes toward risk. Instead, I let market prices speak for themselves as much as possible, extracting from them the implied time series of the factors and their corresponding market prices of risk.

As noted above, one aspect that differentiates this paper from most other work in market-based approach to modeling the yield curve is the explicit effort to provide an economic interpretation to the estimated factors. In particular, I assess the empirical performance of the model in two different ways. First, I look at the model's ability to fit observed moments in the yield curve, both along the time-series and cross-sectional dimensions. Second, I examine the estimated time series of the unobservable factors and check the extent to which they correlate with observable proxies for the economic variables they are meant to represent. For instance, I compare the estimated central tendency series - which is supposed to capture where the short rate is headed - to observed proxies for market participants' short-rate expectations. 


\section{Estimation results}

Given the maximum likelihood estimates of the parameters and latent factors, Figure 2 shows the model's ability to fit the average shape of the yield curve during each of the past nine years. Figure 3 shows actual and model-implied yield curves for the last observation in the sample. Both figures suggest that the two-factor model generally does a good job accounting for both the average level and shape of the curve during the past decade. Figure 4, which shows estimated residuals from equation (28), provides some perspective on how the model captures the time-series variation in the zero-coupon yields. The model residuals are very small, indicating that the Kalman filter was able to ascribe most of the movements in zero-coupon yields to changes in the two estimated factors.

Table 1 summarizes the parameter estimates underlying the model-implied yields shown in Figures 2 through 4 . According to the model, both the short rate and its tendency have a statistically significant degree of mean reversion, the $k$ and $\alpha$ parameters, respectively. The estimated value of $k, 0.42$, implies a half life of about 1.6 years, suggesting that $\theta(t)$ can be thought of as where the short rate will be, in the absence of any unanticipated shocks, over the next few years. The estimated value of $\alpha$-about 0.05 -implies a much slower degree of mean reversion of $\theta(t)$ towards its long-term value, $\beta$, but $\beta$ itself was not precisely estimated by the model and its point estimate seems high. The model did allow me to estimate more precisely the standard errors of the shocks to the short rate and central tendency equations - $v$ and $\eta$ - which, expressed on an annual basis, are estimated to be 84 and 110 basis points, respectively. 
The market price of risk parameters, $\lambda_{r}$ and $\lambda_{\theta}$, were imprecisely estimated in the context of the model. Nonetheless, their point estimates lead to model-implied risk premiums that seem plausible. In particular, I examined risk premiums associated with theoretical short-term interest rate futures contracts, designed to mimic the structure of the three-month eurodollar futures contracts traded at the Chicago Mercantile Exchange, assuming continuous marking to market of futures positions. In the context of the model, and using standard risk-neutral valuation methods, the theoretical time- $t$ futures rate corresponding to the future period $\left[T_{1}, T_{2}\right]$ can be written as

$$
F\left(t, T_{1}, T_{2}\right)=E_{t}^{Q}\left[R\left(T_{1}, T_{2}\right)\right]=J_{0}\left(t, T_{1}, T_{2}\right)+J_{X}\left(t, T_{1}, T_{2}\right) X(t)
$$

where $E_{t}^{Q}[$.$] denotes an expectation taken under the risk-neutral probability$ measure and

$$
\begin{aligned}
J_{0}\left(t, T_{1}, T_{2}\right) & \equiv D_{0}\left(T_{2}-T_{2}\right)+D_{X}\left(T_{2}-T_{1}\right)\left[I-e^{K\left(T_{1}-t\right)}\right] \tilde{\Theta} \\
J_{X}\left(t, T_{1}, T 2\right) & \equiv D_{X}\left(T_{2}-T_{1}\right) e^{-K\left(T_{1}-t\right)}
\end{aligned}
$$

with $\tilde{\Theta} \equiv \Theta-K^{-1}\left[\lambda_{r} v, \lambda_{\theta} \eta\right]^{\prime}$.

Thus, given equations (23) and (30), the risk premium associated with the futures rate $F\left(t, T_{1}, T_{2}\right)$ is given by

$$
\Psi\left(t, T_{1}, T_{2}\right)=D_{X}\left(T_{2}-T_{1}\right)\left[I-e^{K\left(T_{1}-t\right)}\right]\left(-K^{-1}\left[\lambda_{r} v, \lambda_{\theta} \eta\right]^{\prime}\right)
$$

Based on the estimation results, the model suggests that a risk premium of about two basis points per month applies to near-dated interest-rate futures 
contracts. As can be seen in Figure 5, however, the model-implied futures risk premium curve is non-linear in the maturity of the contract, and thus it may be inappropriate to extrapolate the two-basis points per month rule to contracts with longer maturities. For instance, the model-implied risk premium for a two-year ahead three-month futures contract is about 37 basis points, which would amount to roughly 1.5 basis points per month. For a five-year ahead contract, the estimated risk premium is about 57 basis points or .9 basis point per month.

\subsection{Monetary Policy and the Estimated Factors}

The solid lines in the two panels of Figure 6 show the estimated time series for the two unobservable factors and examine their relationship to observed variables. The model specified the first factor as corresponding to a shortterm interest rate, and indeed, as can be seen in the upper panel, the first factor moves very closely in line with observed short-term interest rates, such as the six-month zero-coupon bond yield. Given that the credit quality embedded in the LIBOR/swap curve, from which our zero-coupon yields were derived, is similar to that implicit in the federal funds rate, which is the main monetary policy instrument in the United States, we can think of the first factor as reflecting the current stance of U.S. monetary policy.

The model assumed the second factor to be the central tendency of the short rate, or, according to the estimated mean-reversion parameter for $r(t)$, where the short rate will be in the next three to five years in the absence of future shocks to $r(t)$ and $\theta(t)$. Thus, continuing with the short-rate/monetary policy nexus, the second factor should correspond to market participants' ex- 
pectations of where the monetary policy authorities are expected to take the short rate in the future. Ideally, to assess whether this interpretation of the second factor is a reasonable one, one would need to compare the estimated time series for $\theta(t)$ to market participants' expectations of where the short rate will be, say, over the next three to five years. Such expectations are not directly observable, however, and thus I resort to a commonly used proxy that can be inferred from observed asset prices. In particular, I compared the estimated second factor to short-rate expectations derived from futures rates implied by the three-month eurodollar futures contracts traded at the Chicago Mercantile Exchange.

\section{$5.2 \quad$ Futures-based Interest Rate Expectations}

My primary focus in this part of the paper is to obtain a measure of shortterm interest rate expectations that is independent of the two-factor model. I shall thus step outside that model for the moment and focus on a expectational proxy that is, for the most part, model independent. I start by noting that, given that an eurodollar futures contract's settlement price is based on three-month LIBOR quoted on the settlement date, we can think of the time- $t$ futures rate, $F\left(t, T_{1}, T_{2}\right)$, that corresponds to the three-month period starting in $T_{1}$ and ending in $T_{2}$ as the sum of two components: (i) the conditional expectation of three-month LIBOR that will be quoted at $T_{1}$, $E_{t}\left[R\left(T_{1}, T_{2}\right)\right]$-computed under the objective probability measure and (ii) a risk premium $\mu\left(t, T_{1}, T_{2}\right)$ :

$$
F\left(t, T_{1}, T_{2}\right)=E_{t}\left[R\left(T_{1}, T_{2}\right)\right]+\mu\left(t, T_{1}, T_{2}\right)
$$


Equation (34) reminds us that we cannot simply compare levels of observed futures rates to levels of the estimated second factor. While the former embodies both market participants' expectations and their attitudes toward risk, the model's interpretation of the second factor is that of an expectational variable only.

To start focusing on the expectational component of the futures rate, recall that the results derived in Section 2 imply that

$$
E_{t}\left[R\left(T_{1}, T_{2}\right)\right]=\frac{\int_{T_{1}}^{T_{2}} E_{t}[r(s)] d s}{T_{2}-T_{1}}+\Phi\left(t, T_{1}, T_{2}\right)
$$

which is analogous to (7), except that I am not imposing the expectations hypothesis, i.e., I am potentially allowing for some time variation in the risk premium $\Phi($.$) .$

Assume now that there is a $r^{e}(t)$ such that, for a given future period $\left[T_{1}, T_{2}\right]$, we have

$$
E_{t}[r(s)] \approx r^{e}(t)
$$

for $T_{1} \leq s \leq T_{2}$. In words, (36) assumes that $r$ is expected to revert to a value close to $r^{e}$ by time $T_{1}$ and to remain in the vicinity of that value by time $T_{2}$. Given this assumption, I can write:

$$
E_{t}\left[R\left(T_{1}, T_{2}\right)\right] \approx E_{t}\left[r\left(T_{1}\right)\right]+\Phi\left(t, T_{1}, T_{2}\right)
$$

and thus

$$
F\left(t, T_{1}, T_{2}\right) \approx E_{t}\left[r\left(T_{1}\right)\right]+\Phi\left(t, T_{1}, T_{2}\right)+\mu\left(t, T_{1}, T_{2}\right)
$$


I now follow Kuttner (2001) and assume either that risk premia change little from week to week, or, as implied by the two-factor model, that they are time invariant. Focusing on first differences, I can write:

$$
F\left(t, T_{1}, T_{2}\right)-F\left(t-1, T_{1}, T_{2}\right) \approx E_{t}\left[r\left(T_{1}\right)\right]-E_{t-1}\left[r\left(T_{1}\right)\right]
$$

Thus, we can think of changes in the futures rate $F\left(t, T_{1}, T_{2}\right)$ as a proxy for changes in expectations of where the short rate (monetary policy) will be over some future period $\left[T_{1}, T_{2}\right] .^{7}$

\subsection{Policy expectations and the second factor}

Let us now go back to the two-factor model. I argued that the second factor, $\theta$, can be thought of as reflecting expectations of where $r$ will be over the medium term. Given the results derived in Subsection 5.2, this interpretation of the second factor would imply that changes in $\theta$ should then be highly correlated with changes in appropriately chosen futures rates. To verify this I ran the following regression:

$$
\theta(t)-\theta(t-1)=c_{0}+c_{1}\left[F\left(t, T_{i}, T_{j}\right)-F\left(t-1, T_{i}, T_{j}\right)\right]+w\left(t, T_{i}, T_{j}\right)
$$

where $T_{j}-T_{i}$ corresponds to the three-month period specified in the futures contract; $T_{i}-t$ is the maturity of the futures contract, which varies from

\footnotetext{
${ }^{7}$ An alternative equation describing the relationship between futures rates and policy expectations could be derived by assuming that risk premia follow a random walk. In this case,

$$
F\left(t, T_{1}, T_{2}\right)-F\left(t-1, T_{1}, T_{2}\right) \approx E_{t}\left[r\left(T_{1}\right)\right]-E_{t-1}\left[r\left(T_{1}\right)\right]+\epsilon(t)
$$

where $\epsilon(t)$ follows a white noise process.
} 
a few days to several years, and $w\left(t, T_{i}, T_{j}\right)$ is the corresponding regression residual.

The upper panel of Table 2 summarizes the results of regressions based on futures contracts with maturities ranging from one to nine years. ${ }^{8}$ Because the data for some contracts with maturities longer than five years are only available starting in the early to mid-1990s, however, these regressions are based on a shorter sample than the one used for the estimation of the two-factor model. The results suggest a strong link between changes in the estimated second factor and changes in short rate expectations derived from the eurodollar futures rates. The estimated $R^{2}$ s are higher than 0.80 in most cases, especially for the regressions involving futures rates corresponding to contracts maturing in three to six years, a result that is consistent with the estimated speed of mean reversion of the short rate in the two-factor model. Moreover, the estimated slopes of most regressions are not far from one, suggesting a nearly one-to-one relationship between changes in the second factor and movements in this market-based measure of the expected future stance of monetary policy. The estimated intercepts were all found to be statistically insignificant. To take into account the possibility that the regression errors may be non-i.i.d.-for instance, because futures rates may embed a time varying risk premium - the t-statistics reported in the table are consistent with possible heteroskedasticity and autocorrelation of regression residuals (Newey and West, 1987).

The lower panel of Table 2 focuses on futures contracts expiring in three

\footnotetext{
${ }^{8}$ Futures contracts expiring in ten years are also traded at the CME, but were not included in this analysis because of their more limited data availability.
} 
to six years, a time horizon that is more in line with the estimated halflife of 1.6 years for the short rate process. These contracts also have the advantage of having a longer historical record, in that the data go back to 1989. The results confirm the findings reported for the regressions based on the shorter sample. They suggest a high degree of comovement between the estimated second factor and medium-term policy expectations inferred from eurodollar futures rates, with a one percentage point increase in the threeyear eurodollar futures rate being associated with an increase in the second factor of similar magnitude.

\subsection{Interpreting historical movements in the yield curve}

We can use the model to interpret observed movements in the yield curve. For instance, as can be seen in Figure 6, the model suggests that the substantial easing in monetary policy in the early 1990s was initially taken by market participants as a temporary phenomenon. In particular, $\theta(t)$ hardly moved from its level in 1989 until late 1992, leading to a considerable steepening of the yield curve during that period. The downward revision in monetary policy expectations in 1992 would prove to be short-lived, however, as the model interprets movements in the yield curve in the second half of 1993 as being driven by a sharp upward revision in the expected path of short rates. Indeed, even as the Federal Reserve started tightening policy in February 1994, the estimated time series for $\theta(t)$ would suggest that market participants were expecting even higher levels of short-term rates farther down the road. The gap between the short rate and its model-implied central tendency was considerably narrower during the rest of the 1990s, implying 
that the model would characterize the observed stance of policy during that time as being roughly consistent with the expected stance of policy. For instance, although $\theta(t)$ started rising considerably in early 1999, the series of tightening actions that got under way later that year would again bring the actual policy stance broadly in line with the model-implied longer-term policy stance.

\subsection{Level, slope, and curvature}

Figure 7 shows how the model would characterize the response of the yield curve to unanticipated one percentage point increases in each of the factors. As shown in the upper panels, a positive shock to the short rate alone would result in a substantial flattening of the yield curve, with a one-percentage point rise in the short rate being associated with a nearly 90 basis point narrowing in the spread between the six-month and the thirty-year zerocoupon bond yields. The intuition behind this result is straightforward: The rise in the short rate is viewed as temporary and thus the response of longerdated yields to the short-rate shock decreases with maturity.

The middle panels show the estimated yield curve's response to an unexpected one-percentage increase in the central tendency of the short rate. Here the model suggests two effects: First, the yield curve steepens considerably, as short rates are anticipated to rise in the future. A second, more subtle, effect regards the curvature of the yield curve: The shock is felt more strongly around the ten-year sector of the curve because the model posits that $\theta(t)$ will eventually revert back to its long-run value.

Lastly, the lower panels of Figure 7 show the effect of simultaneous posi- 
tive shocks to $r(t)$ and $\theta(t)$ : The entire yield curve moves higher in response to these shocks. Thus, the model captures the so-called level, slope, and curvature effects that are commonly referred to in the term structure literature.

\section{Concluding Remarks}

I estimated a two-factor term structure model that fits the data very well both across time and maturity. The analysis carried out in this paper differs from other work in the affine term structure literature in two main ways. First, in setting up the model, I proposed an economic interpretation to each factor, as opposed to the usual practice of labeling them according to their effects on the level, slope, and curvature of the yield curve. Second, I checked explicitly whether the proposed interpretation of the factors is born out by the data. It is. The first and second latent factors essentially correspond to the current and expected stance of monetary policy, in that they move nearly one-to-one with short-term LIBOR and medium-term eurodollar futures rates, respectively.

I illustrated how the model can be used to characterize movements in the yield curve since the late 1980s. The model provides a plausible account of how expectations about future monetary policy have helped shape the yield curve over this period. I also used the model to quantify the effects of changes in each factor on the yield curve, showing they can be directly mapped into level, slope, and curvature effects.

In this paper, I provided an expectations-hypothesis interpretation of the model, highlighting the fact that a common form of the hypothesis generally 
does not ensure the absence of arbitrage opportunities. I did not, however, address a vast literature that focuses on empirical tests of the expectations hypothesis - see, e.g., Dai and Singleton (2002) and references therein. I consider this a topic for another paper. In future work, I also intend to extend the model to allow for changing risk premiums, as the imprecise estimates of the market prices of risks reported in this paper suggest a possible misspecification of the time-invariant risk premiums.

Lastly, it is worth noting that, because the model discussed in this paper was build upon an arbitrage-free framework, its uses go well beyond the important topic of unveiling the relationship between monetary policy and the yield curve. For instance, the model can be used in the pricing and hedging of a large array of more complex instruments, such as bond options, swaptions, mortgage-backed securities, and credit derivatives. 


\section{References}

Ang, A. and M. Piazzesi, "A No-Arbitrage Vector Autoregression of Term Structure Dynamics with Macroeconomic and Latent Variables," 2002. Working paper, UCLA.

Babbs, S. and B. Nowman, "Kalman Filtering of Generalized Vasicek Term Structure Models," Journal of Financial and Quantitative Analysis, 1999, 34, 115-30.

Balduzzi, P., S. Das, and S. Foresi, "The Central Tendency: A Second Factor in Bond Yields," The Review of Economics and Statistics, 1998, $80,62-72$.

Bjork, T., Arbitrage Theory in Continuous Time, Oxford University Press, 1998.

Cox, J., J. Ingersoll, and S. Ross, "A Re-examination of Traditional Hypothesis about the Term Structure of Interest Rates," The Journal of Finance, 1981, 36, 769-99.

Dai, Q. and K. Singleton, "Specification Analysis of Affine Term Structure Models," The Journal of Finance, 2000, 55, 1943-78.

and _ _ "Expectations Puzzles, Time-varying Risk Premia, and Affine Models of the Term Structure," Journal of Financial Economics, 2002, 63, 415-41. 
Evans, C. and D. Marshall, "Monetary Policy and the Term Structure of Nominal Interest Rates: Evidence and Theory," Carnegie-Rochester Conference Series on Public Policy, 1998, 49, 53-111.

Kuttner, K., "Monetary Policy Surprises and Interest Rates: Evidence from the Fed Funds Futures Market," Journal of Monetary Economics, $2001,47,523-44$.

Litterman, R. and J. Scheinkman, "Common Factors Affecting Bond Returns," The Journal of Fixed Income, June 1991, pp. 54-61.

Newey, W. and K. West, "A Simple Positive Semi-Definite, Heteroskedasticity and Autocorrelation Consistent Covariance Matrix," Econometrica, 1987, 55, 703-8.

Piazzesi, M., "Macroeconomic Jump Effects and the Yield Curve," 2001. Working paper, UCLA.

Vasicek, O., "An Equilibrium Characterization of the Term Structure," Journal of Financial Economics, 1977, 5, 177-88. 
Table 1 -Estimation Results ${ }^{a}$

\begin{tabular}{lcc}
\hline \hline Parameter & Estimated value & Standard error \\
\hline & & \\
$k$ & 0.4186 & 0.0039 \\
$\alpha$ & 0.0458 & 0.0015 \\
$\beta$ & 0.0838 & 0.0588 \\
$\eta$ & 0.0110 & 0.0003 \\
$v$ & 0.0084 & 0.0002 \\
$\lambda_{r}$ & 40.9367 & 38.951 \\
$\lambda_{\theta}$ & 0.1273 & 23.389 \\
$h_{1 / 2}$ & 0.1310 & 0.0086 \\
$h_{1}$ & 0.0001 & 0.0003 \\
$h_{2}$ & 0.1254 & 0.0123 \\
$h_{3}$ & 0.1122 & 0.0116 \\
$h_{5}$ & 0.0608 & 0.0029 \\
$h_{7}$ & 0.0001 & 0.0067 \\
$h_{10}$ & 0.0671 & 0.0021 \\
& & \\
\hline \hline
\end{tabular}

${ }^{a}$ Estimation period: 1989 to 2001. The data are weekly (667 observations). The model was estimated using zero-coupon yields derived from LIBOR and swap rates for the following maturities: six-months, one, two, three, five, seven, and ten years. $h_{i}$ is the standard error of the residuals of the measurement equation associated with a zero-coupon bond with $i$ years to maturity. 
Table 2 - The Second Factor and Monetary Policy Expectations ${ }^{a}$

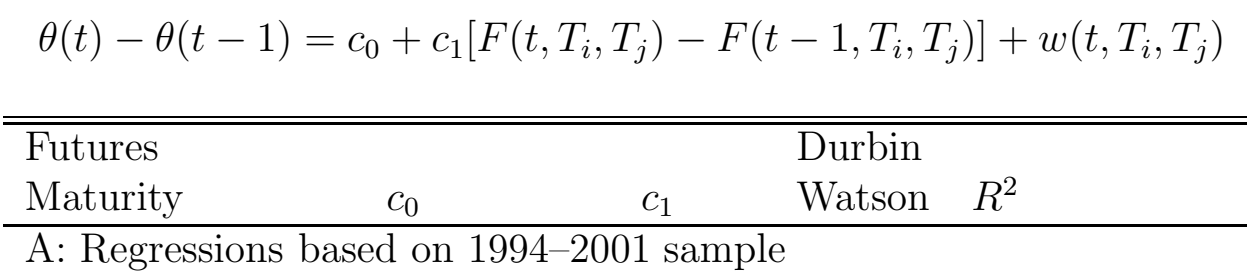

$\begin{array}{lllll}\text { two years } & .00(.08) & 1.06(24.6) & 1.91 & .78 \\ \text { three years } & .00(.10) & 1.22(45.2) & 2.08 & .88 \\ \text { four years } & .00(.35) & 1.26(54.3) & 2.29 & .90 \\ \text { five years } & .00(.53) & 1.26(54.3) & 2.28 & .89 \\ \text { six years } & .00(.53) & 1.25(49.8) & 2.22 & .87 \\ \text { seven years } & .00(.44) & 1.21(42.01 & 2.11 & .83 \\ \text { eight years } & .00(.40) & 1.16(34.8) & 1.93 & .78 \\ \text { nine years } & .00(.39) & 1.09(28.7) & 1.83 & .76\end{array}$

\begin{tabular}{lllll}
\hline B: Regressions based on 1989-2001 sample & \\
& & & \\
three years & $.00(.93)$ & $1.10(36.0)$ & 2.13 & .78 \\
four years & $.00(.73)$ & $1.18(45.5)$ & 2.27 & .82 \\
five years & $.00(.35)$ & $1.23(51.1)$ & 2.41 & .86 \\
six years & $.00(.52)$ & $1.25(49.9)$ & 2.27 & .87 \\
\hline \hline
\end{tabular}

${ }^{a}$ The data are weekly. Heteroskedasticity- and autocorrelation-consistent t-statistics shown in parenthesis (Newey and West, 1987). 
Figure 1

Swap-implied zero-coupon yields

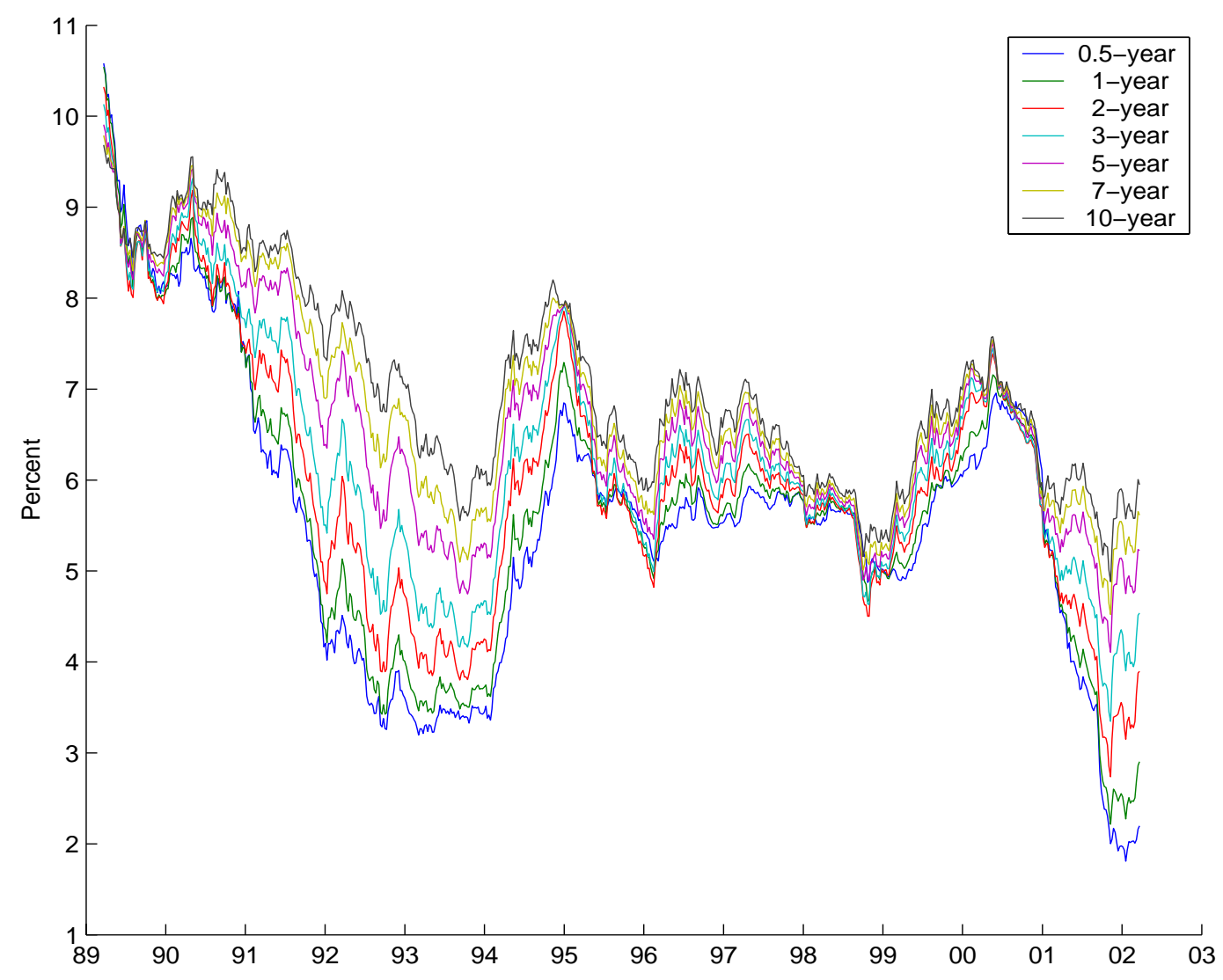


Figure 2

Actual (solid) \& Model-implied (dotted) Yield Curves
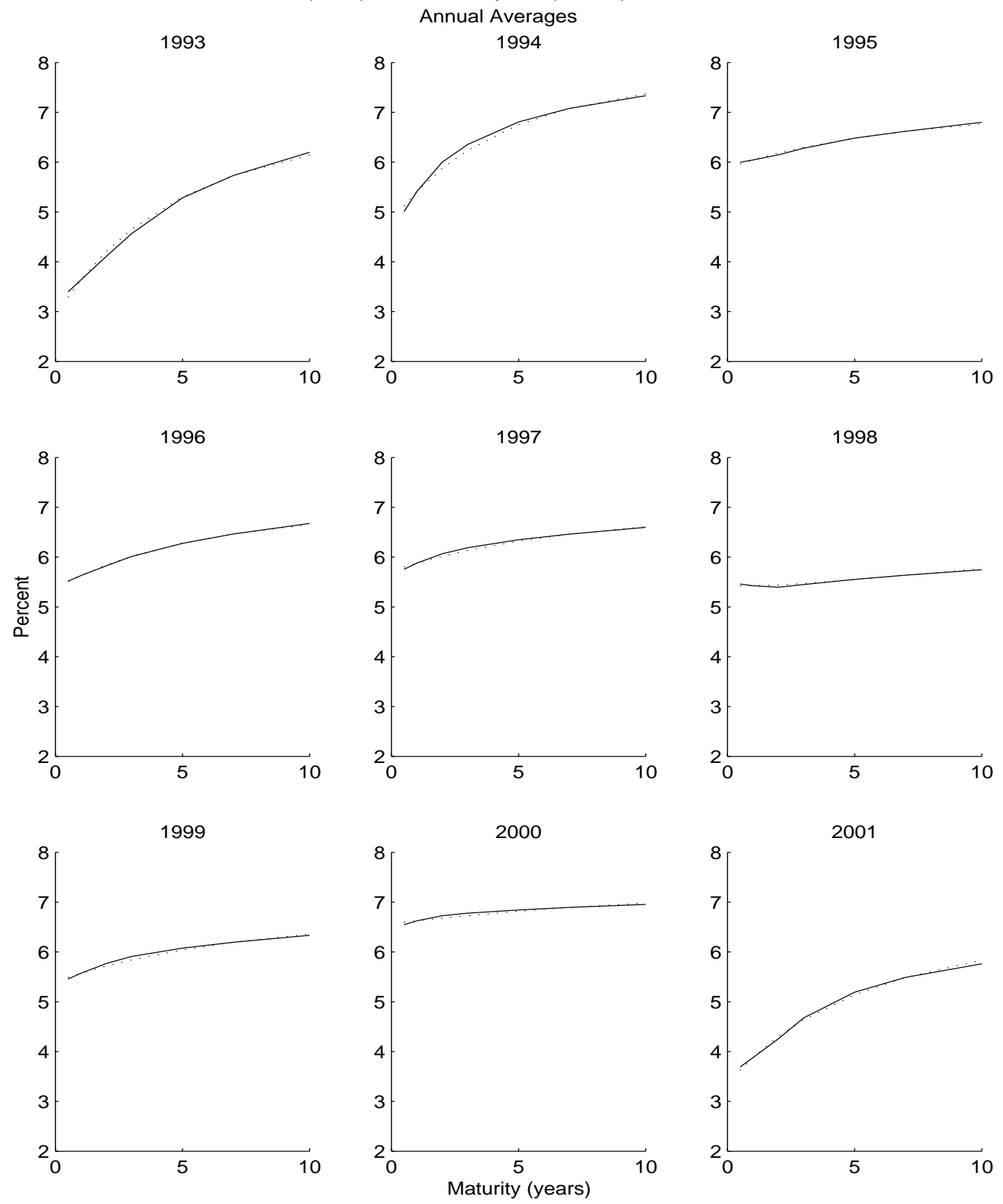
Figure 3

Swap-implied zero yield curve, week ended Mar 22, 2002

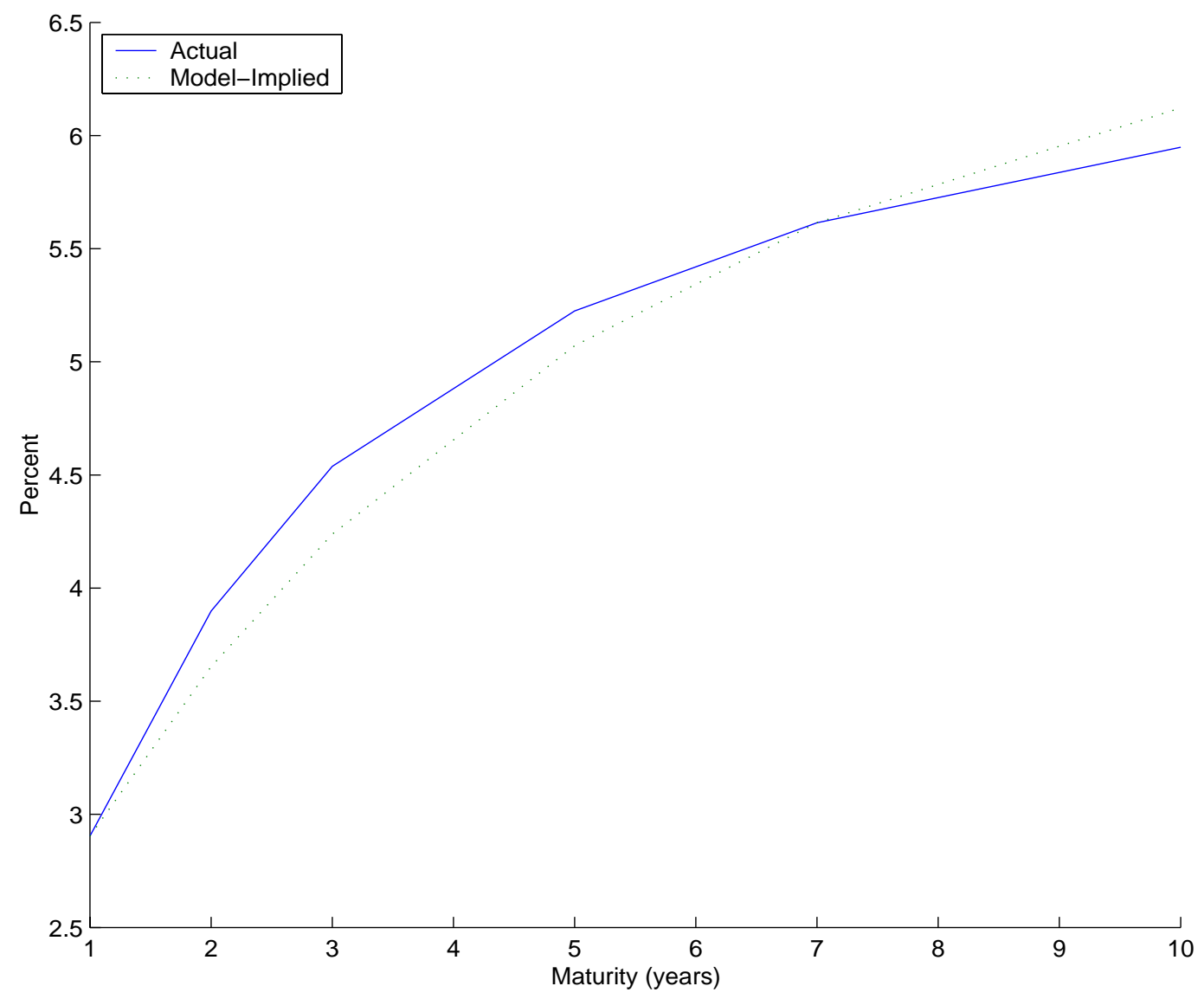


Figure 4

Measurement Equation Residuals
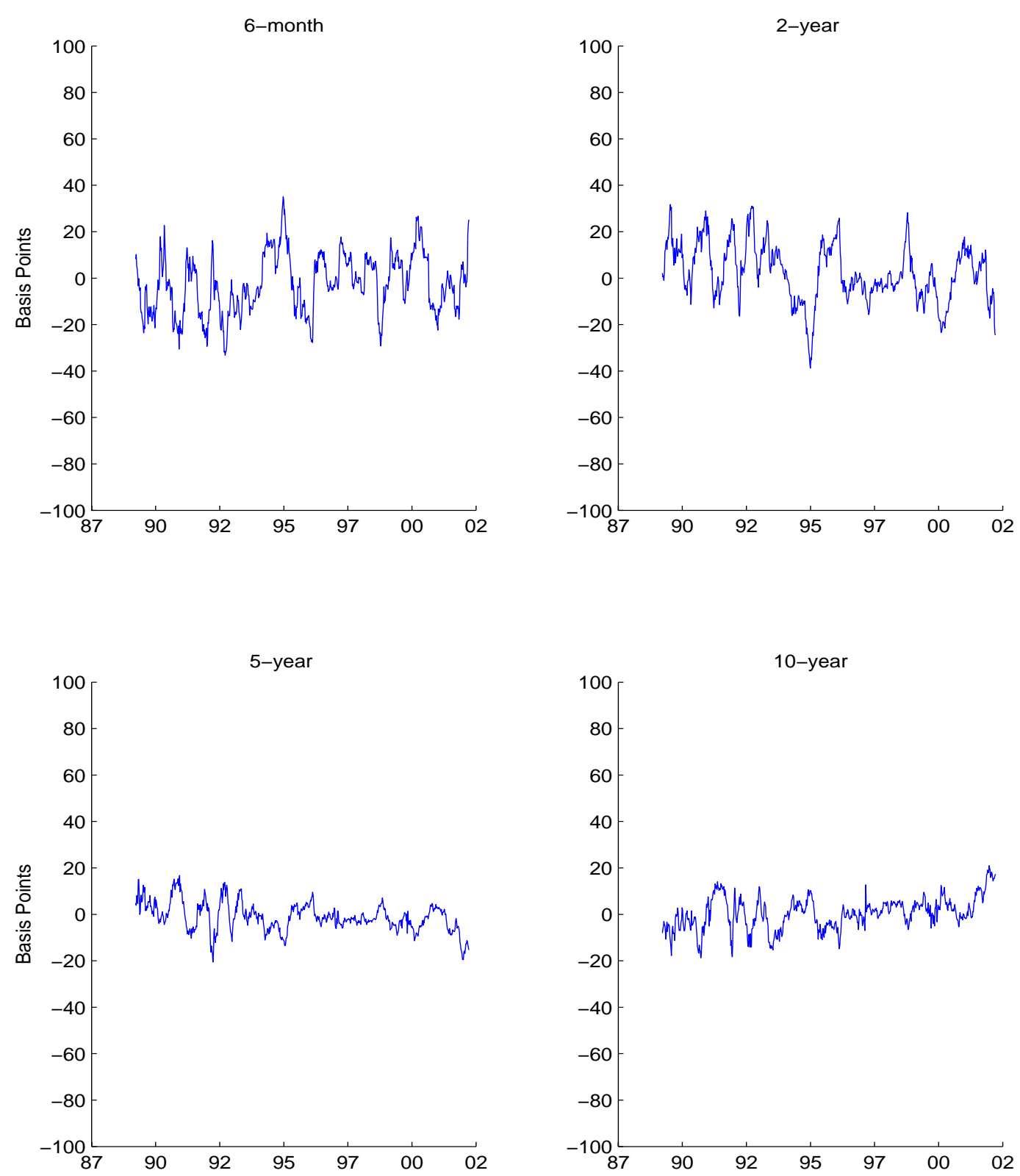
Figure 5

Term Structure of Risk Premiums Associated with

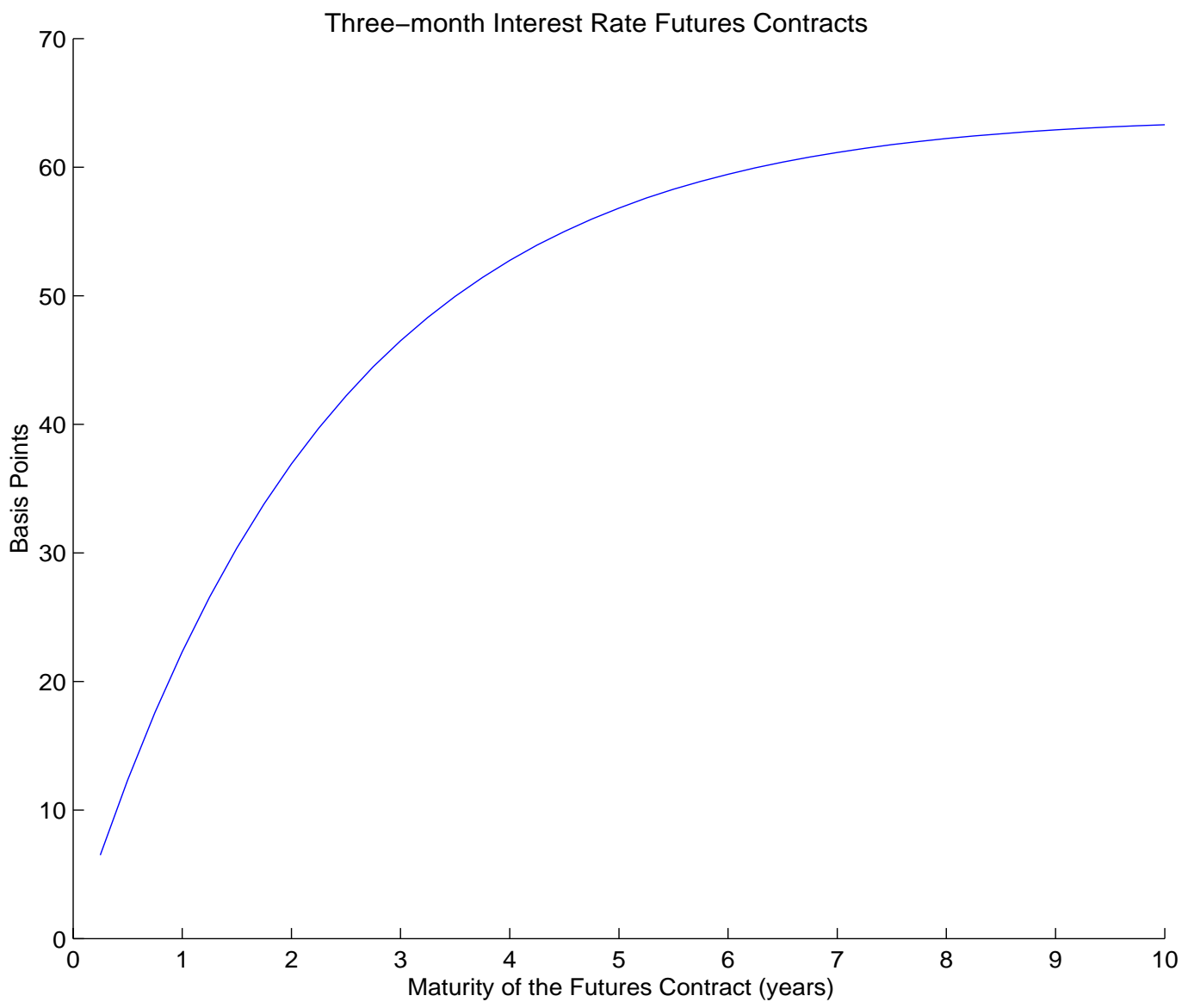


Figure 6
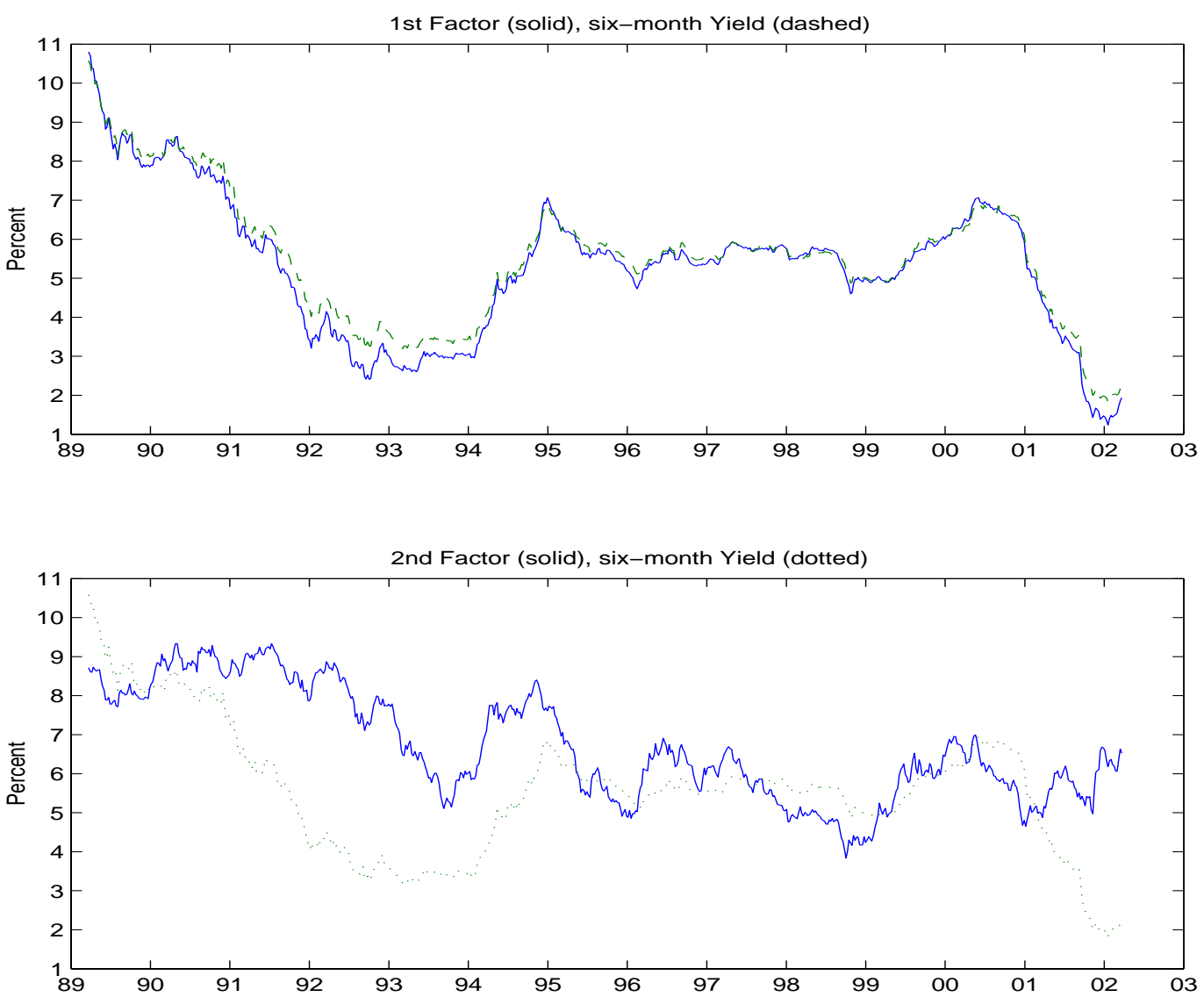
Figure 7

Level, Slope, and Curvature Effects
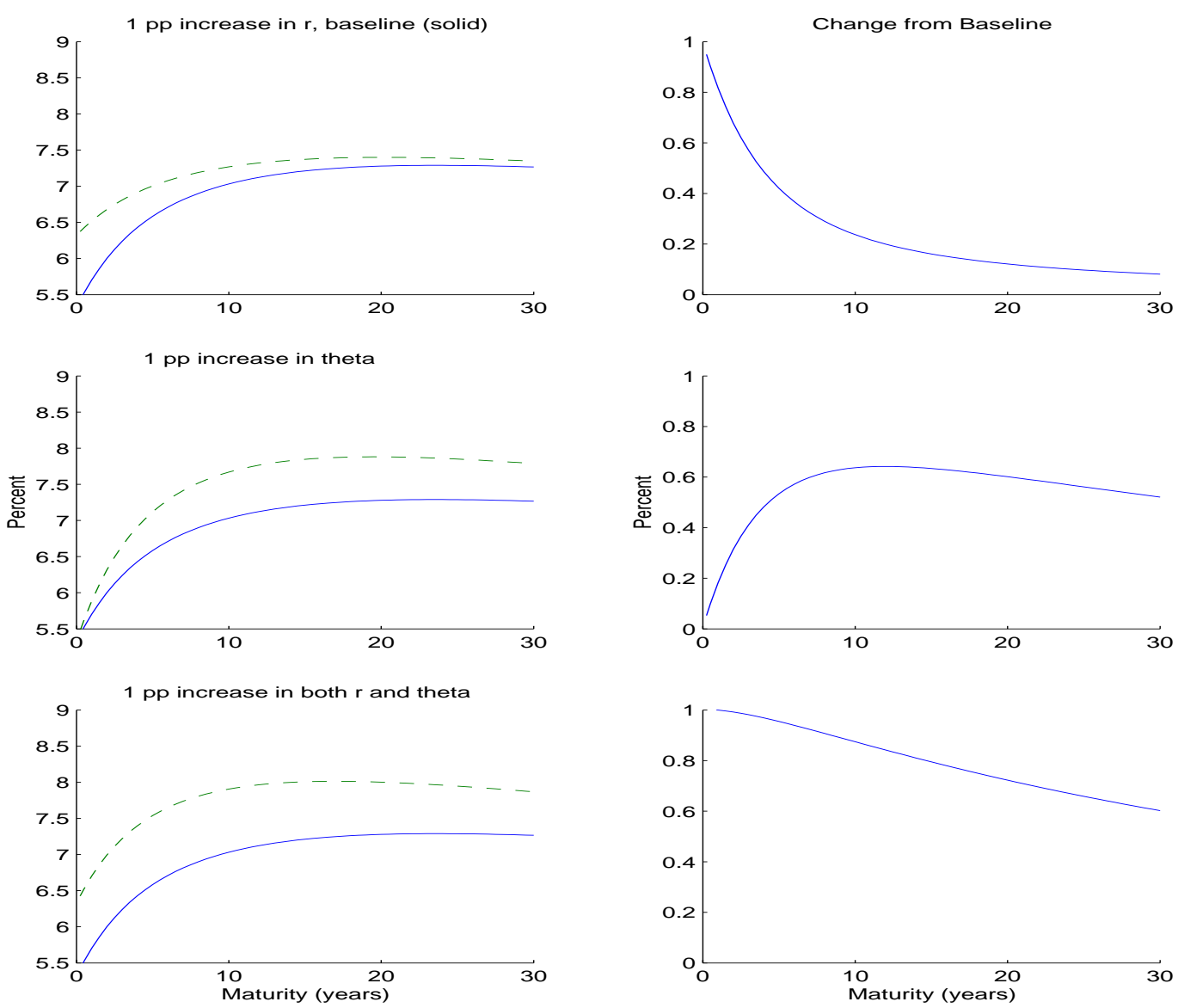